\title{
Survey of Overnight Academic Hospitalist Supervision of Trainees
}

\author{
Jeanne M. Farnan, MD, MHPE ${ }^{1 *}$, Alfred Burger, MD², Romsai T. Boonayasai, MD, MPH³, Luci Leykum, MD, MS ${ }^{4}$, \\ Rebecca Harrison, MD ${ }^{5}$, Julie Machulsky, BA ${ }^{6}$, Vikas Parekh, $\mathrm{MD}^{7}$, Bradley A. Sharpe, MD ${ }^{8}$, \\ Anneliese M. Schleyer, MD, MHA ${ }^{9}$, Vineet M. Arora, MD, MAPP; ${ }^{10}$ for the SGIM Housestaff Oversight Subcommittee
}

\begin{abstract}
${ }^{1}$ Department of Medicine, Section of Hospital Medicine, The University of Chicago, Chicago, Illinois; '2Department of Medicine, Section of General Internal Medicine, Beth Israel Medical Center, Albert Einstein College of Medicine, New York, New York; ${ }^{3}$ Department of Medicine, Section of General Internal Medicine, Johns Hopkins University School of Medicine, Baltimore, Maryland; "Department of Medicine, South Texas Veterans Health Care Systems, University of Texas Health Science Center San Antonio, San Antonio, Texas; ${ }^{5}$ Department of Medicine, Division of Hospital Medicine, Oregon Health \& Science University, Portland, Oregon; 'SSociety of General Internal Medicine, Alexandria, Virginia; 'Department of Medicine, Division of General Medicine, University of Michigan Medical School, Ann Arbor, Michigan; ${ }^{\circ}$ Department of Medicine, Division of Hospital Medicine, University of California-San Francisco, San Francisco, California; ${ }^{9}$ Department of Medicine, Division of General Internal Medicine, University of Washington, Seattle, Washington; ${ }^{10}$ Department of Medicine, Section of General Internal Medicine.
\end{abstract}

In 2003, Accreditation Council for Graduate Medical Education (ACGME) announced the first in a series of guidelines related to the residency training. The most recent recommendations include explicit recommendations regarding the provision of on-site clinical supervision for trainees of internal medicine. To meet these standards, many internal medicine residency programs turned to hospitalist programs to fill that need. However, much is unknown about the current relationships between hospitalist and residency programs, specifically with regard to supervisory roles and supervision policies. We aimed to describe how academic hospitalists currently supervise housestaff during the on-call, or overnight, period and hospitalist program leader their perceptions of how these new policies would impact trainee-hospitalist interactions. Journal of Hospital Medicine 2012;7:521-523. (c) 2012 Society of Hospital Medicine
In 2003, the Accreditation Council for Graduate Medical Education (ACGME) announced the first in a series of guidelines related to the regulation and oversight of residency training. ${ }^{1}$ The initial iteration specifically focused on the total and consecutive numbers of duty hours worked by trainees. These limitations began a new era of "shift work" in internal medicine residency training. With decreases in housestaff admitting capacity, clinical work has frequently been offloaded to "non-teaching" or attending-only services, increasing the demand for hospitalists to fill the void in physician-staffed care in the hospital. ${ }^{2,3}$ Since the implementation of the 2003 ACGME guidelines and a growing focus on patient safety, there has been increased study of, and call for, oversight of trainees in medicine; among these was the 2008 Institute of Medicine report, ${ }^{4}$ calling for $24 / 7$ attending-level supervision. The updated ACGME requirements, ${ }^{5}$ effective July 1, 2011, mandate enhanced on-site supervision of trainee physicians. These new regulations not only define varying levels of supervision for

*Address for correspondence and reprint requests: Jeanne M. Farnan, MD, MHPE, Department of Medicine and Pritzker School of Medicine, The University of Chicago, 5841 S Maryland Ave, MC 2007, AMB W216, Chicago, IL 60637; Telephone: 773-834-8904; Fax: 773-834-2238; E-mail: jfarnan@medicine.bsd.uchicago.edu

Additional Supporting Information may be found in the online version of this article.

Received: February 17, 2012; Revised: June 5, 2012; Accepted: June 18,2012

2012 Society of Hospital Medicine DOI 10.1002/jhm.1961

Published online in Wiley Online Library (Wileyonlinelibrary.com). trainees, including direct supervision with the physical presence of a supervisor and the degree of availability of said supervisor, they also describe ensuring the quality of supervision provided. ${ }^{5}$ While continuous attending-level supervision is not yet mandated, many residency programs look to their academic hospitalists to fill the supervisory void, particularly at night. However, what specific roles hospitalists play in the nighttime supervision of trainees or the impact of this supervision remains unclear. To date, no study has examined a broad sample of hospitalist programs in teaching hospitals and the types of resident oversight they provide. We aimed to describe the current state of academic hospitalists in the clinical supervision of housestaff, specifically during the overnight period, and hospitalist perceptions of how the new ACGME requirements would impact trainee-hospitalist interactions.

\section{METHODS}

The Housestaff Oversight Subcommittee, a working group of the Society of General Internal Medicine (SGIM) Academic Hospitalist Task Force, surveyed a sample of academic hospitalist program leaders to assess the current status of trainee supervision performed by hospitalists. Programs were considered academic if they were located in the primary hospital of a residency that participates in the National Resident Matching Program for Internal Medicine. To obtain a broad geographic spectrum of academic hospitalist programs, all programs, both university and community-based, in 4 states and 2 metropolitan regions 
were sampled: Washington, Oregon, Texas, Maryland, and the Philadelphia and Chicago metropolitan areas. Hospitalist program leaders were identified by members of the Taskforce using individual program websites and by querying departmental leadership at eligible teaching hospitals. Respondents were contacted by e-mail for participation. None of the authors of the manuscript were participants in the survey.

The survey was developed by consensus of the working group after reviewing the salient literature and included additional questions queried to internal medicine program directors. ${ }^{6}$ The 19 -item SurveyMonkey instrument included questions about hospitalists' role in trainees' education and evaluation. A Likert-type scale was used to assess perceptions regarding the impact of on-site hospitalist supervision on trainee autonomy and hospitalist workload $(1=$ strongly disagree to $5=$ strongly agree). Descriptive statistics were performed and, where appropriate, $t$ test and Fisher's exact test were performed to identify associations between program characteristics and perceptions. Stata SE was used (STATA Corp, College Station, TX) for all statistical analysis.

\section{RESULTS}

The survey was sent to 47 individuals identified as likely hospitalist program leaders and completed by 41 individuals $(87 \%)$. However, 7 respondents turned out not to be program leaders and were therefore excluded, resulting in a $72 \%(34 / 47)$ survey response rate.

The programs for which we did not obtain responses were similar to respondent programs, and did not include a larger proportion of community-based programs or overrepresent a specific geographic region. Twenty-five $(73 \%)$ of the 34 hospitalist program leaders were male, with an average age of 44.3 years, and an average of 12 years post-residency training (range, 5-30 years). They reported leading groups with an average of 18 full-time equivalent (FTE) faculty (range, 3-50 persons).

\section{Relationship of Hospitalist Programs With the Residency Program}

The majority $(32 / 34,94 \%)$ of respondents describe their program as having traditional housestaff-hospitalist interactions on an attending-covered housestaff teaching service. Other hospitalists' clinical roles included: attending on uncovered (non-housestaff services; 29/34, 85\%); nighttime coverage (24/34, $70 \%)$; attending on consult services with housestaff $(24 / 34,70 \%)$. All respondents reported that hospitalist faculty are expected to participate in housestaff teaching or to fulfill other educational roles within the residency training program. These educational roles include participating in didactics or educational conferences, and serving as advisors. Additionally, the faculty of $30(88 \%)$ programs have a formal evalua- tive role over the housestaff they supervise on teaching services (eg, members of formal housestaff evaluation committee). Finally, 28 (82\%) programs have faculty who play administrative roles in the residency programs, such as involvement in program leadership or recruitment. Although $63 \%$ of the corresponding internal medicine residency programs have a formal housestaff supervision policy, only $43 \%$ of program leaders stated that their hospitalists receive formal faculty development on how to provide this supervision to resident trainees. Instead, the majority of hospitalist programs were described as having teaching expectations in the absence of a formal policy.

Twenty-one programs $(21 / 34,61 \%)$ described having an attending hospitalist physician on-site overnight to provide ongoing patient care or admit new patients. Of those with on-site attending coverage, a minority of programs $(8 / 21,38 \%)$ reported having a formal defined supervisory role of housestaff trainees for hospitalists during the overnight period. In these 8 programs, this defined role included a requirement for housestaff to present newly admitted patients or contact hospitalists with questions regarding patient management. Twenty-four percent $(5 / 21)$ of the programs with nighttime coverage stated that the role of the nocturnal attending was only to cover the non-teaching services, without housestaff interaction or supervision. The remainder of programs $(8 / 21,38 \%)$ describe only informal interactions between housestaff and hospitalist faculty, without clearly defined expectations for supervision.

\section{Perceptions of New Regulations and Night Work}

Hospitalist leaders viewed increased supervision of housestaff both positively and negatively. Leaders were asked their level of agreement with the potential impact of increased hospitalist nighttime supervision. Of respondents, $85 \%(27 / 32)$ agreed that formal overnight supervision by an attending hospitalist would improve patient safety, and $60 \%(20 / 33)$ agreed that formal overnight supervision would improve traineehospitalist relationships. In addition, $60 \%(20 / 33)$ of respondents felt that nighttime supervision of housestaff by faculty hospitalists would improve resident education. However, approximately 40\% (13/33) expressed concern that increased on-site hospitalist supervision would hamper resident decision-making autonomy, and $75 \%(25 / 33)$ agreed that a formal housestaff supervisory role would increase hospitalist work load. The perception of increased workload was influenced by a hospitalist program's current supervisory role. Hospitalists programs providing formal nighttime supervision for housestaff, compared to those with informal or poorly defined faculty roles, were less likely to perceive these new regulations as resulting in an increase in hospitalist workload (3.72 vs $4.42 ; P=0.02$ ). In addition, hospitalist programs with a formal nighttime role were more likely to 
identify lack of specific parameters for attending-level contact as a barrier to residents not contacting their supervisors during the overnight period (2.54 vs 3.54; $P=0.03$ ). No differences in perception of the regulations were noted for those hospitalist programs which had existing faculty development on clinical supervision.

\section{DISCUSSION}

This study provides important information about how academic hospitalists currently contribute to the supervision of internal medicine residents. While academic hospitalist groups frequently have faculty providing clinical care on-site at night, and often hospitalists provide overnight supervision of internal medicine trainees, formal supervision of trainees is not uniform, and few hospitalists groups have a mechanism to provide training or faculty development on how to effectively supervise resident trainees. Hospitalist leaders expressed concerns that creating additional formal overnight supervisory responsibilities may add to an already burdened overnight hospitalist. Formalizing this supervisory role, including explicit role definitions and faculty training for trainee supervision, is necessary.

Though our sample size is small, we captured a diverse geographic range of both university and community-based academic hospitalist programs by surveying group leaders in several distinct regions. We are unable to comment on differences between responding and non-responding hospitalist programs, but there does not appear to be a systematic difference between these groups.

Our findings are consistent with work describing a lack of structured conceptual frameworks in effectively supervising trainees, ${ }^{7,8}$ and also, at times, nebulous expectations for hospitalist faculty. We found that the existence of a formal supervisory policy within the associated residency program, as well as defined roles for hospitalists, increases the likelihood of positive perceptions of the new ACGME supervisory recommenda- tions. However, the existence of these requirements does not mean that all programs are capable of following them. While additional discussion is required to best delineate a formal overnight hospitalist role in trainee supervision, clearly defining expectations for both faculty and trainees, and their interactions, may alleviate the struggles that exist in programs with illdefined roles for hospitalist faculty supervision. While faculty duty hours standards do not exist, additional duties of nighttime coverage for hospitalists suggests that close attention should be paid to burn-out. ${ }^{9}$ Faculty development on nighttime supervision and teaching may help maximize both learning and patient care efficiency, and provide a framework for this often unstructured educational time.

\section{Acknowledgements}

The research reported here was supported by the Department of Veterans Affairs, Veterans Health Administration, Health Services Research and Development Service (REA 05-129, CDA 07-022). The views expressed in this article are those of the authors and do not necessarily reflect the position or policy of the Department of Veterans Affairs.

\section{References}

1. Philibert I, Friedman P, Williams WT. New requirements for resident duty hours. JAMA. 2002;288:1112-1114.

2. Nuckol T, Bhattacharya J, Wolman DM, Ulmer C, Escarce J. Cost implications of reduced work hours and workloads for resident physicians. N Engl J Med. 2009;360:2202-2215.

3. Horwitz L. Why have working hour restrictions apparently not improved patient safety? BMJ. 2011;342:d1200.

4. Ulmer C, Wolman DM, Johns MME, eds. Resident Duty Hours: Enhancing Sleep, Supervision, and Safety. Washington, DC: National Academies Press; 2008.

5. Nasca TJ, Day SH, Amis ES Jr; for the ACGME Duty Hour Task Force. The new recommendations on duty hours from the ACGME Task Force. N Engl J Med. 2010;363.

6. Association of Program Directors in Internal Medicine (APDIM) Survey 2009. Available at: http://www.im.org/toolbox/surveys/SurveyDataand Reports/APDIMSurveyData/Documents/2009_APDIM_summary_web. pdf. Accessed on July 30, 2012.

7. Kennedy TJ, Lingard L, Baker GR, Kitchen L, Regehr G. Clinical oversight: conceptualizing the relationship between supervision and safety. $J$ Gen Intern Med. 2007;22(8):1080-1085.

8. Farnan JM, Johnson JK, Meltzer DO, et al. Strategies for effective oncall supervision for internal medicine residents: the SUPERB/SAFETY model. J Grad Med Educ. 2010;2(1):46-52.

9. Glasheen J, Misky G, Reid M, Harrison R, Sharpe B, Auerbach A. Career satisfaction and burn-out in academic hospital medicine. Arch Intern Med. 2011;171(8):782-785. 